\title{
Remote Data Transmission Technology Based on BeiDou Satellite Navigation Sensor System Onboard Ship
}

\author{
Xueqiang Zhuang, ${ }^{1,2}$ Yiqun Xu, ${ }^{1,2}$ Yali Gao, ${ }^{1,2 *}$ Guanglou Sun, ${ }^{1,2}$ \\ Tianjin Lin, ${ }^{1,2}$ and Christopher Chun Ki Chan ${ }^{3 * *}$ \\ ${ }^{1}$ School of Marine Engineering, Jimei University, Xiamen 361021, China \\ ${ }^{2}$ Provincial Key Laboratory of Naval Architecture \& Ocean Engineering, Xiamen 361021, China \\ ${ }^{3}$ Department of Information Management, Chaoyang University of Technology, 413310, Taiwan
}

(Received July 20, 2020; accepted November 16, 2020)

Keywords: BeiDou Navigation Satellite System, data remote transmission technology, STC89C52RC chip, ship

Currently, commercially available systems for ship navigation and remote data transmission technology are maritime satellites and BeiDou satellite systems, the global positioning system (GPS), and mobile communication technology. Among these, mobile communication technology and GPS can only perform ship navigation and lack data transmission functionality. In this research, we analyze technical characteristics of several common remote data transmission methods and we develop and design an in-ship remote data transmission system based on the BeiDou Satellite Navigation Sensor System (BDS). We use an STC89C52RC data acquisition microcontroller chip, which is connected with an HS215 BeiDou communication position module via an RS232 standard serial port that allows us to capture, collect, parse, package, and send ship data. We adopt the shore-based ship standard NMEA protocol to receive, parse, display, and save data. Our system is tested in a simulation and with a real ship, and the results are in line with expectations (low cost, reliable, and safe). We believe that our system will be economically and socially beneficial by solving maritime problems and also beneficial to the safety and emergency rescue of marine vessels.

\section{Introduction}

With the rapid development of the international and domestic shipping economy, the flow of maritime traffic and the size of marine vessels are increasing. As a result, there is an increased risk of pollution from ship accidents. In order to ensure the safety of maritime navigation, improve the efficiency of maritime traffic flow, and reduce the risk of ship-related pollution, it is imperative to study ship data transmission technology in order to monitor ship safety in real time in an efficient and effective manner.

Maritime satellite communication solutions are often costly and only used in times of distress. Ship remote data transmission technology without the use of satellites is also capable of the real-time monitoring, technical support, and basic management of ships through a shipping company or vessel traffic service (VTS). Zhu et al. performed a comprehensive study of

\footnotetext{
*Corresponding author: e-mail: yali_gao77@outlook.com

** Corresponding author: e-mail: christopherckchan@cyut.edu.tw

https://doi.org/10.18494/SAM.2021.3038
} 
existing BeiDou Satellite Navigation Sensor System (BDS)-related global positioning system (GPS) software and solutions, ${ }^{(1)}$ in which a quality analysis of BeiDou-2 as a global navigation satellite positioning system was performed, as well as methods of noise reduction and a discussion of its positioning software package. These operators have two main functions: to provide accurate and timely positioning information for navigation and to ensure stable and smooth ship-toship and ship-to-shore communication. ${ }^{(2)}$ At present, there are six main commercially available communication technologies that can be used for the remote transmission of ship data, and their technical characteristics are shown in Table $1 .^{(3-8)}$

We perform a comparative analysis on these technical characteristics and develop a BDSbased remote ship data transmission technology. BDS is a system that has many advantages, which include wide coverage, low cost, secure transmission, and real-time monitoring of the ship's path trajectory as well as relaying safety status information. At the same time, an offshore VTS can use the transmitted data provided by a ship to provide offshore support for the on-board crew. This information enables better and effective decision-making and crew management as well as provides crucial information for emergency ship rescue in the event of a ship incident. The use of this technology better guarantees safe ship navigation via effective communication and ensures the preservation of maritime life and property.

\section{Introduction to BeiDou Navigation Satellite System}

The BeiDou Navigation Satellite System is a satellite navigation system independently built and operated in China, which can provide all-weather and all-day services including basic navigation (positioning, speed measurement, timing), global short message communication, international emergency search and rescue, and other services for Asian and global users. ${ }^{(9-12)}$ BDS consists of three segments: a space segment, a ground segment, and a user segment. Its working principle is shown in Fig. 1.

Table 1

Comparison of ship remote data transmission technologies.

\begin{tabular}{|c|c|c|}
\hline $\begin{array}{l}\text { Communication } \\
\text { technology }\end{array}$ & Communication features & Scope of application \\
\hline $\begin{array}{l}\text { Universal mobile } \\
\text { communication } \\
\text { network }\end{array}$ & $\begin{array}{l}\text { Low communication cost and large network bandwidth; small } \\
\text { coverage and low network security. }\end{array}$ & $\begin{array}{l}\text { Suitable for inland rivers } \\
\text { and offshore ships. }\end{array}$ \\
\hline $\begin{array}{l}\text { Automatic } \\
\text { identification } \\
\text { system (AIS) }\end{array}$ & $\begin{array}{l}\text { Can directly realize the speed measurement navigation function } \\
\text { of ship positioning and monitoring and carry out information } \\
\text { broadcasting; high service cost, seriously affected by electromagnetic } \\
\text { environment interference, communication distance is limited. }\end{array}$ & $\begin{array}{l}\text { Suitable for inland rivers } \\
\text { and offshore ships. }\end{array}$ \\
\hline $\begin{array}{l}\text { Maritime satellite } \\
\text { system }\end{array}$ & $\begin{array}{l}\text { Can provide data link for ocean-going ships; high cost, low } \\
\text { communication bandwidth, maximum speed is only } 492 \mathrm{Kbps} \text {. }\end{array}$ & $\begin{array}{l}\text { Suitable for global } \\
\text { navigation ships. }\end{array}$ \\
\hline $\begin{array}{l}\text { Spread spectrum } \\
\text { communication } \\
\text { technology }\end{array}$ & $\begin{array}{l}\text { Strong concealment and good anti-interference performance; } \\
\text { the system uses a wide frequency band and has a limited data } \\
\text { transmission rate and distance. }\end{array}$ & $\begin{array}{l}\text { Suitable for inland rivers } \\
\text { and offshore ships. }\end{array}$ \\
\hline GPS & Accurate positioning and strong anti-interference ability. & $\begin{array}{l}\text { Only applicable to ship } \\
\text { positioning, unable to } \\
\text { achieve communication. }\end{array}$ \\
\hline BDS & $\begin{array}{l}\text { All-weather, all-day, high-precision positioning; short message } \\
\text { communication has low cost and high security. }\end{array}$ & $\begin{array}{l}\text { Suitable for global } \\
\text { navigation ships. }\end{array}$ \\
\hline
\end{tabular}




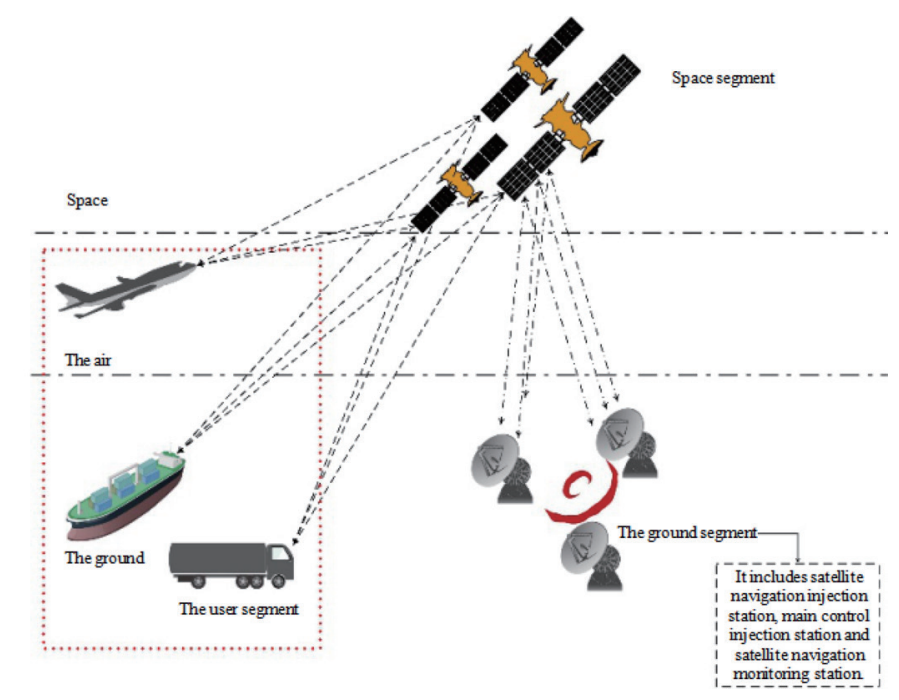

Fig. 1. (Color online) Schematic diagram of BDS working principle.

Since BDS was developed after the adoption of other systems, it includes many improvements and advancements upon other systems. The advantages of BDS for ship-to-ship and ship-to-shore telecommunication applications include:

(1) High-precision positioning, navigation, timing, and other functions, and the ability to conduct bidirectional short message remote data communication between the BeiDou navigation terminal system and users or between BeiDou terminal users and the data reception management center.

(2) High transmission speed, reliable transmission, strong anti-jamming capability, secure transmission, and wide coverage via short message communication. It is also stable and accurate in areas covered by the marine mobile communication network.

(3) With regard to automated ships, important parameters related to the automation of ships can be monitored in real time, as well as providing important data collection and consistent information sharing.

(4) BDS user terminals are relatively light and cost-effective in terms of technology and service.

(5) BDS has a large information capacity with high concurrency of data, stable real-time communication, and comprehensive reception of ship-to-shore interactive data.

\section{Overall Design of Ship Remote Data Transmission Technology Based on BDS}

The main task of ship remote data transmission technology is to transmit the location information collected by sensors and the ship's basic data such as temperature, pressure, humidity, and liquid level to onshore monitoring personnel through the BeiDou communication link in a nondestructive manner. Onshore management personnel then feed back the relevant commands and instructions to the ship. On the basis of the basic data of ships and BDS, we design and develop an entire data transmission system from aspects such as data collection, transmission, and data reception onshore. Its overall design structure is divided into two parts: the data collection and transmission device onshore and onboard. 


\subsection{Hardware design of shipboard data collection and transmission device}

The shipboard data collection and transmission device adopts a modular design, mainly including a shipboard microcontroller processing module, a BeiDou satellite system positioning and communication processing module, a shipboard data acquisition sensor module, and a shipboard power control part. Its basic principle and structure are shown in Fig. 2.

\subsubsection{Microcontroller processing module}

The microcontroller module mainly performs data acquisition. This acquisition module involves data collection and analysis of the ship's position, as well as packaging and transmitting the processed information to a desired receiver. The acquisition module's purpose is to process data with a low power while providing strong anti-jamming capability in a simple structure at a low cost. On the basis of these features, an STC89C52RC microcontroller is selected as our proposed system's ship data acquisition module microcontroller.

The design features of the STC89C52RC microcontroller are as follows:

(1) The serial port is connected to the BeiDou communication and positioning module, which is designed to send position setting instructions. This port activates the BeiDou communication and positioning module and enables it to receive the current positioning signal of the ship in real time, and subsequently feed real-time position information of the ship back to the shipborne microcontroller module.

(2) Once the shipboard microcontroller module receives an interrupt signal, the interrupt program begins to receive, analyze, and store the corresponding position of the ship with a timer to an array queue. The queue waits for a signal from the sensor data acquisition module, which calculates the cruising speed and combines the information into a data package ready for transmission.

(3) The shipboard microcontroller module program sets a $60 \mathrm{~s}$ timer interrupt in the sending queue. When the timer interrupt is triggered, the array queue packages the data of the corresponding ship position information in accordance with BeiDou short message remote transmission protocol format, and sends the packaged information via a serial port to a BeiDou communication module.

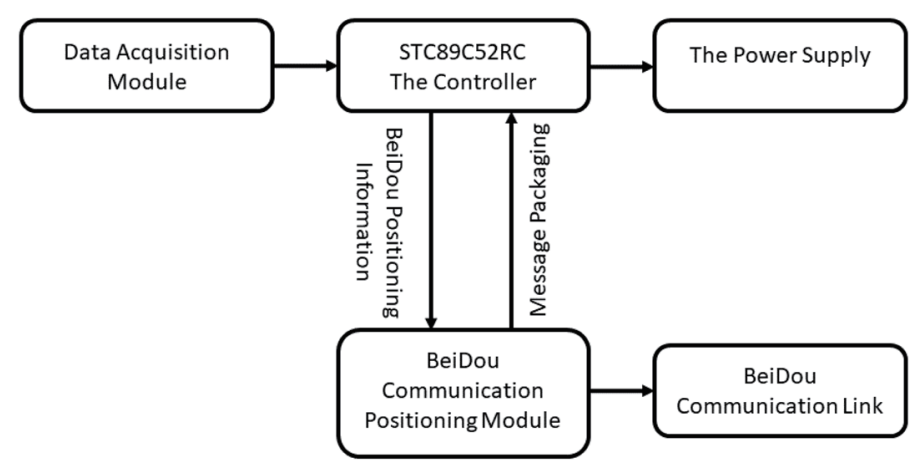

Fig. 2. Schematic diagram of shipboard data transceiver device. 


\subsubsection{BeiDou communication positioning module}

The design features of the BeiDou communication positioning module are as follows:

(1) Receive positioning and setting instructions of the ship's microcontroller, and transmit the ship's current position to the ship-borne microcontroller module through a serial port. The ship's current position data includes the ship's real-time position, date, time, longitude, latitude, and elevation.

(2) Receive the ship communication data sent by the ship-borne microcontroller through the serial port. The ship communication data includes the ship's current position information, temperature, pressure, humidity, liquid level, and other relevant information that is collected by the data acquisition sensor module.

On the basis of the requirements of BeiDou communication module procedures, we choose a Nanjing Tian Hong Chen S215 module as the BeiDou positioning communication module. This module is modified in accordance with BeiDou dedicated high-performance lownoise communication protocols. The entire module consists of a radiodetermination satellite service (RDSS) RF transceiver communication chip, a 5-W-output high-power RF amplifier communication module, a BeiDou terminal dedicated RDSS positioning communication baseband circuit, and a BD2 Bl/GPS L1 miniaturized navigation positioning module. All of these components allow RDSS communication and RNSS navigation. ${ }^{(13)}$

\subsubsection{Serial port communication}

The BeiDou positioning communication module is connected with the ship microcontroller through a serial port to transmit data. After analysis and comprehensive consideration, the standard serial port RS232 connection is selected for the proposed system. An HS215 communication module operates through an RS232 standard serial port that is connected with the microcontroller module. In order for the system to function, the BeiDou communication sends information through the TXD pin. The position information is then sent to the microcontroller module serial port at the receiving end through an RXD pin, which reads the position information and performs data analysis. This data analysis consists of reading the position information and sensor data information and then packaging the information based on BeiDou short message communication protocol.

\subsection{Software design of shipboard data collection and transmission device}

In this paper, the shipboard data transceiver device uses $\mathrm{C}$ language as a programming language to realize the software design, and its functional program design is shown in Fig. 3. The BeiDou HS215 module interface protocol meets the NMEA protocol, and its instruction format is shown in Table 2. The statements of the BeiDou communication protocol include \$BDSMS, \$BDPOS, \$SMSXX, \$POSXX, and other statements, among which \$POSXX is the statement of the controller module to obtain ship position information, including the date, time, longitude and latitude coordinates, and elevation. After receiving the ship position information, 


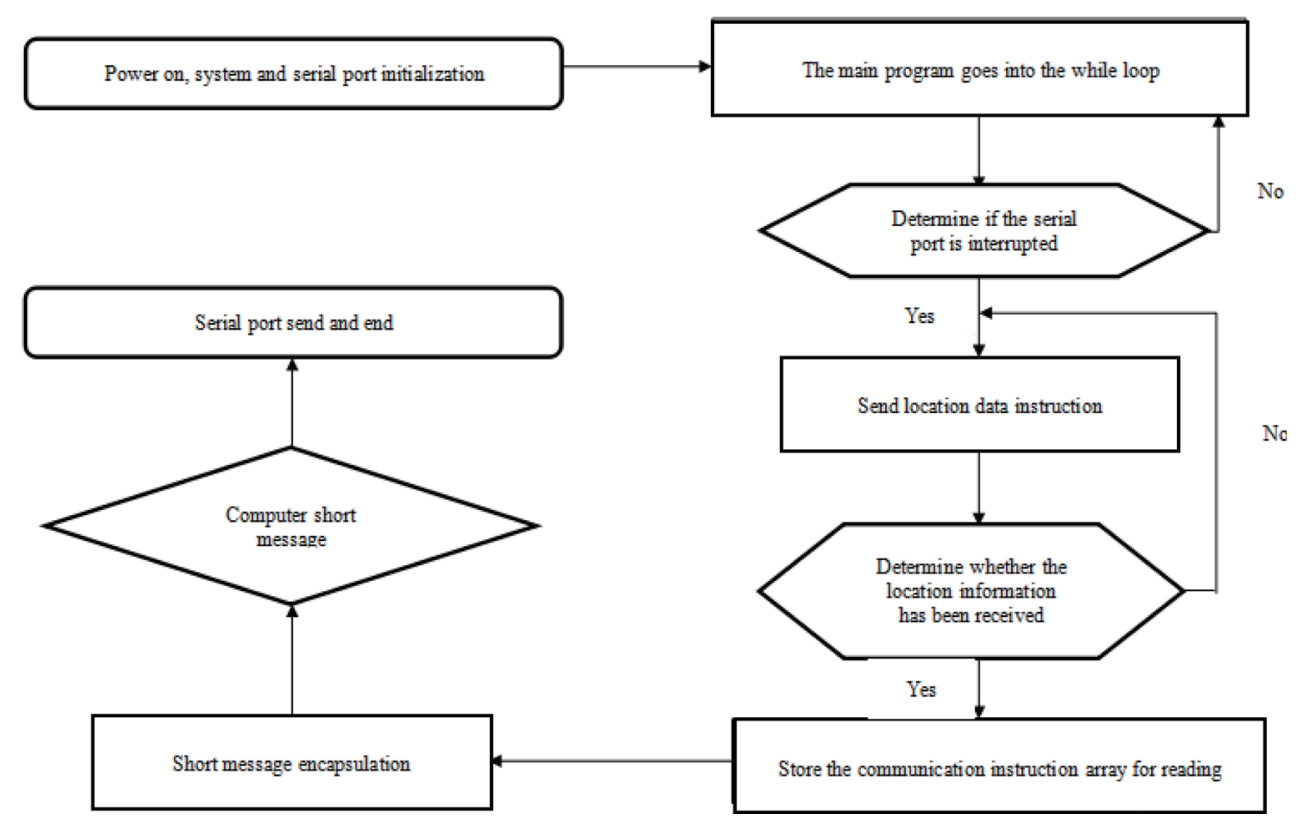

Fig. 3. Overall flow chart of shipboard software.

Table 2

Instruction format of BeiDou HS215 module.

\begin{tabular}{lccccc}
\hline Data fields & Instruction names & Length of data & User ID & Message content & Checksum \\
\hline Number of bytes per field & 6 & 2 & 3 & Custom & 1
\end{tabular}

the microcontroller module parses and processes the collected data and ship position data, and stores them in the short message remote communication sending array of BDSMS for packaging and sending according to the BeiDou communication protocol. A short message is based on user requirements, code numbers, and the use of ASCII characters, as well as GB2312 Chinese character encoding. We defined a message protocol that consists of instruction names, length, sender ID, receiver ID, latitude and longitude, time, temperature, flow, pressure, liquid level, inclination, concentration, and checksum.

\subsection{Shore-based ship data receiving procedure}

After the BeiDou receiver receives the message from the sending end, it accesses the upper computer acquisition program through the RS232 serial port feedback instruction \$SMSXX. SerialPort name, baud rate, stop bit, data bit, check bit, and other SerialPort parameters are sent through the background event of the software SerialPort button, and the data acquisition function is realized by SerialPort control. First, a string variable "buffer" is defined as the content of the string cache, and then it is converted from hexadecimal to decimal. Then, the string interception function is called to intercept the cached array data for communication information and data parsing. After the program receives the data, it is automatically saved to 
the local TXT folder to provide data support for the subsequent interface development program. The specific software design process is shown in Fig. 4.

\section{Ship-based Test of Remote Data Transmission System}

\subsection{Ship and time measured}

This test was carried out using the ship Yude of Jimei University (Fig. 5), which is now operated by COSCO Shipping Company. The ship has a total length of $199.90 \mathrm{~m}$, a width of $32.26 \mathrm{~m}$, a depth of $18.00 \mathrm{~m}$, and a total load of $64000 \mathrm{t}$. It can accommodate 138 teachers and students at the same time. The main engine model of Yude is DMD MAN B\&W5S60ME-C8.2, with a continuous power of $6842.5 \mathrm{~kW}$ and a speed of $84.3 \mathrm{rpm}$. The main engine sensor includes a speed probe and a throttle load sensor. The boiler model is LYF2.0/364-0.6, the heating area is

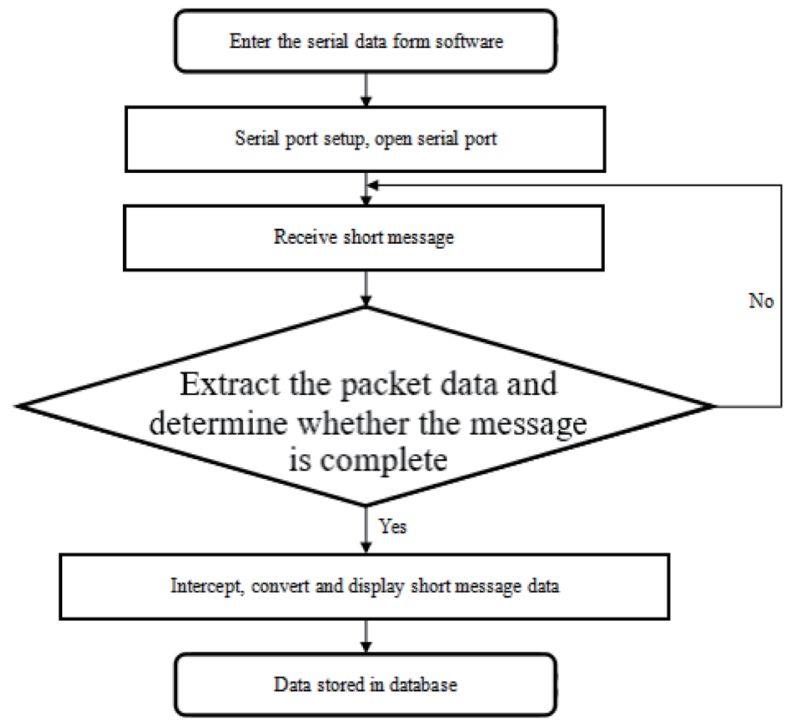

Fig. 4. BeiDou short message receiving program.

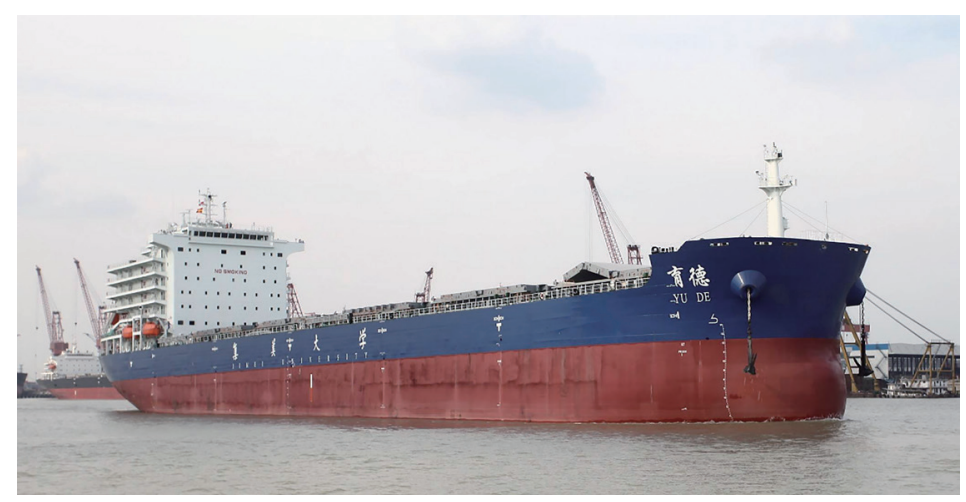

Fig. 5. (Color online) Appearance of Yude. 
$364 \mathrm{~m}^{2}$, the evaporation capacity is $2.0 \mathrm{t} / \mathrm{h}$, and the evaporation pressure is $0.6 \mathrm{MPa}$. The boiler sensors include a pressure sensor, water level sensor, salinometer, and oil content detector. The test took place on June 14 and 19, 2020.

\subsection{Data acquisition and transmission test at the shipboard end}

In the experiment, program-burning software by STC-ISP (a single-chip program-burning software designed for the STC series microcontroller) and the software program of the shipboard data transmitting and receiving device were installed in the STC89C52RC experimental microcontroller. An RS232 serial line was used to connect the BeiDou module and the STC89C52RC experimental microcontroller, a USB line was used to connect a microcontroller unit (MCU) and PC, and a $220 \mathrm{~V}$ alternating current was used as the experimental power. Then, the test software of the single-chip microcomputer was opened to set the basic settings for serial port communication. After the single-chip microcomputer was powered on, the test software of the single-chip microcomputer serial port was opened to simulate and test the BeiDou position information feedback.

\subsection{Shore-based receiving tests for ship data}

In the test of the shore-based ship data receiving application program, household $220 \mathrm{~V}$ AC power was also selected as the experimental power supply, and a 9-pin RS232 to the USB serial port line was used to connect the BeiDou receiver and PC. The message received in this test includes the date, time, longitude and latitude, rudder angle, steam pressure of the boiler, water level of the boiler, inclination angle, rotating speed of the host, throttle load of the host, and other information. The test results are shown in Fig. 6.

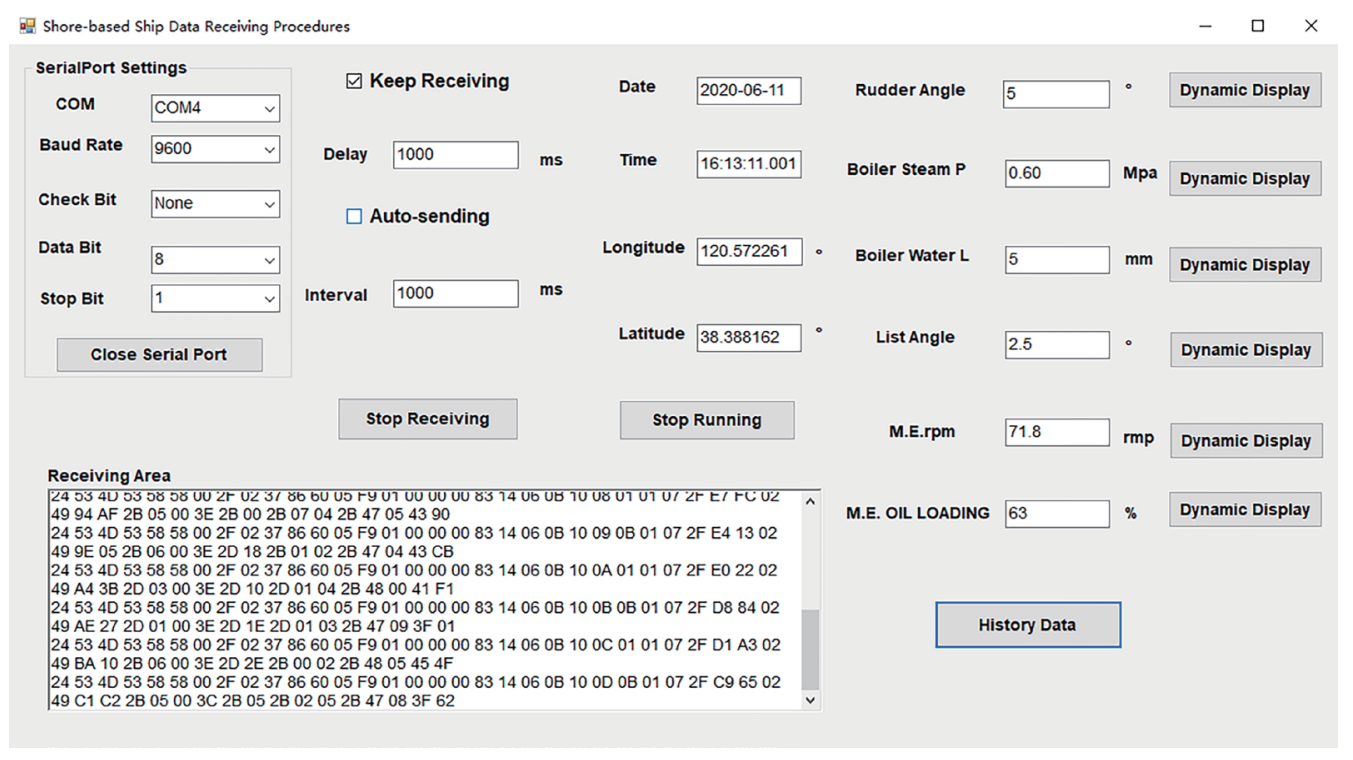

Fig. 6. (Color online) Test results of shipboard data receiving interface. 


\subsection{Test results of data transmission onboard ship}

The output BeiDou position information of Yude in this test is shown in Fig. 7. The test results verified the smooth operation of the whole system. When the system is working normally, the SCM collects the ship positioning information and transmits it to the shorebased data receiving center in real time, so as to realize the real-time monitoring of the ship's trajectory.

In addition, we carried out real-time data transmission tests of safety parameters such as rudder angle, boiler steam pressure, boiler water level, inclination angle, host speed, and throttle load in two different running states of the ship at the dock and at sea, for which the test results are respectively shown in Figs. 8 and 9. When the ship is docked at the dock, the main engine

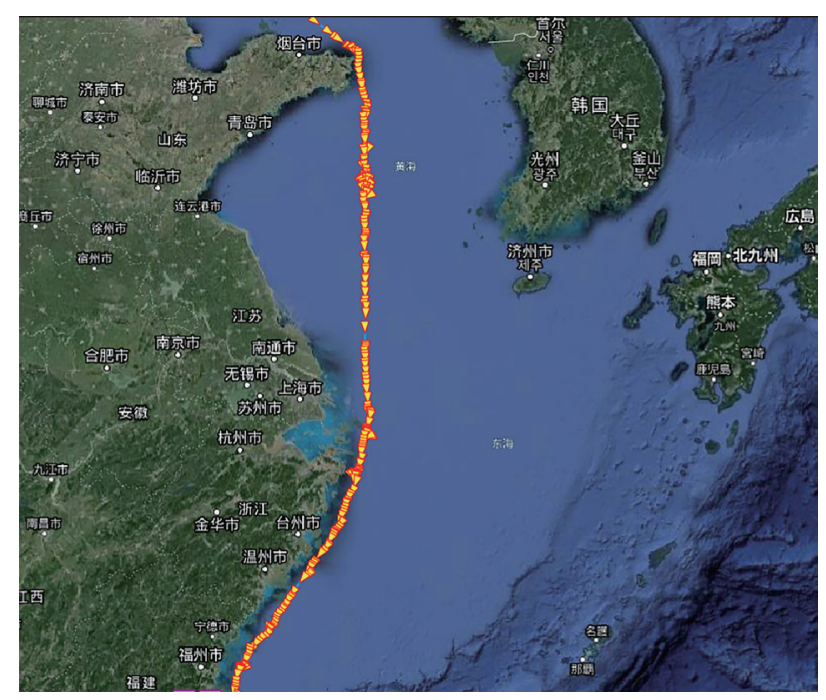

Fig. 7. (Color online) Real-time position information transmission of Yude.

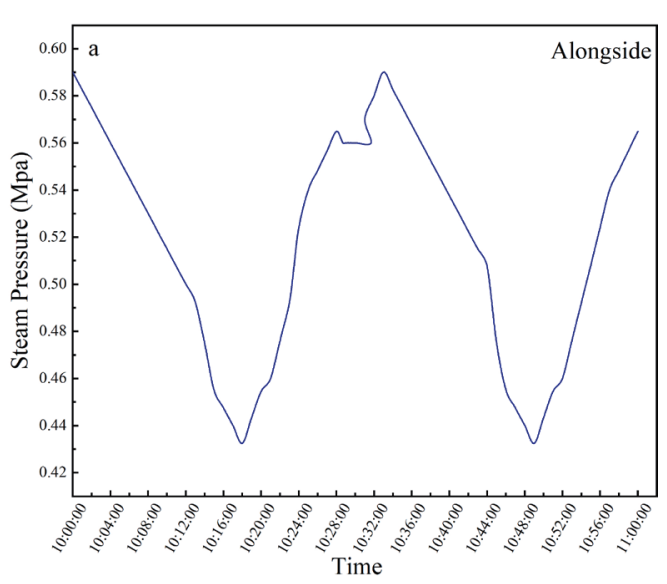

(a)

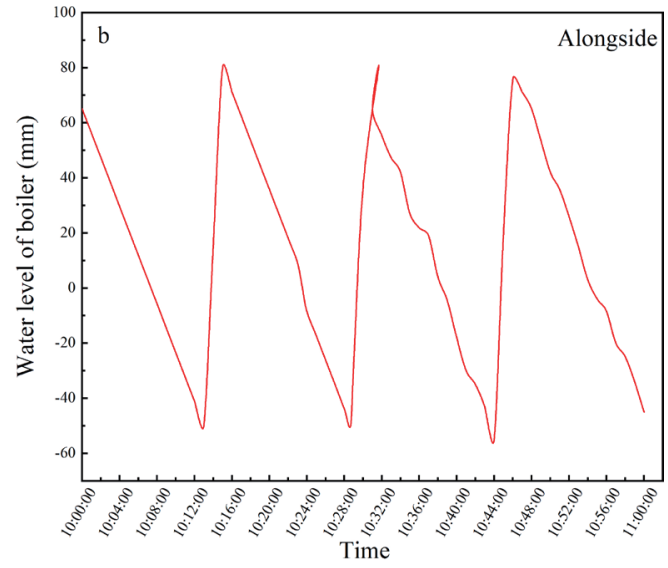

(b)

Fig. 8. (Color online) Simulated parameters and output results of ship berthing state: (a) boiler steam pressure; (b) boiler water level. 


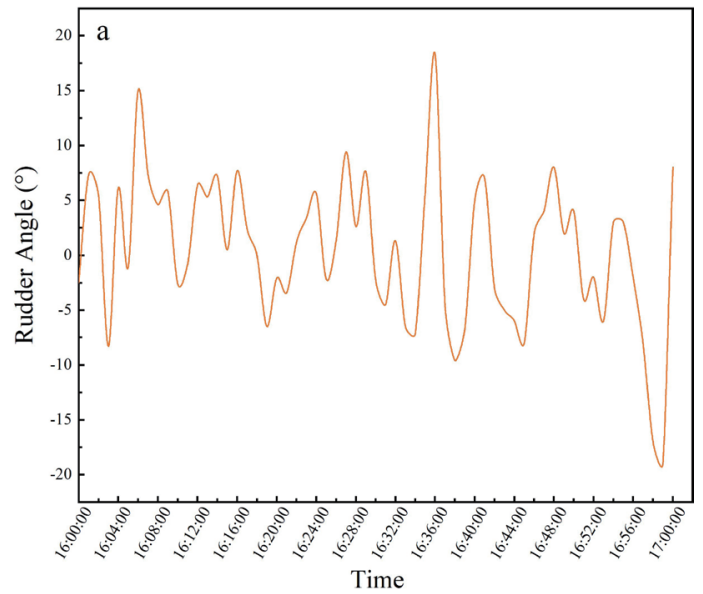

(a)

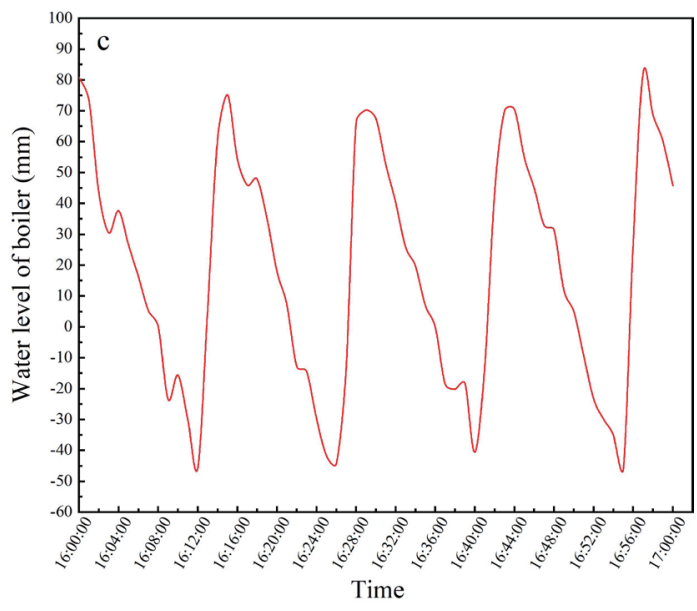

(c)

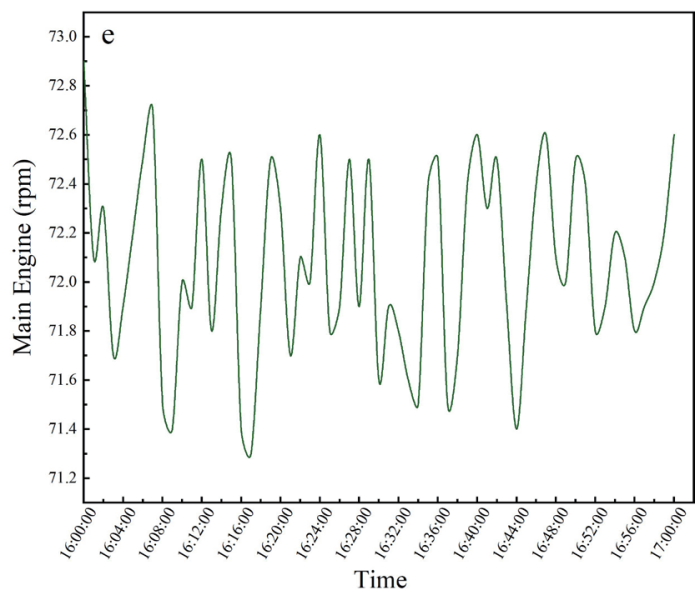

(e)

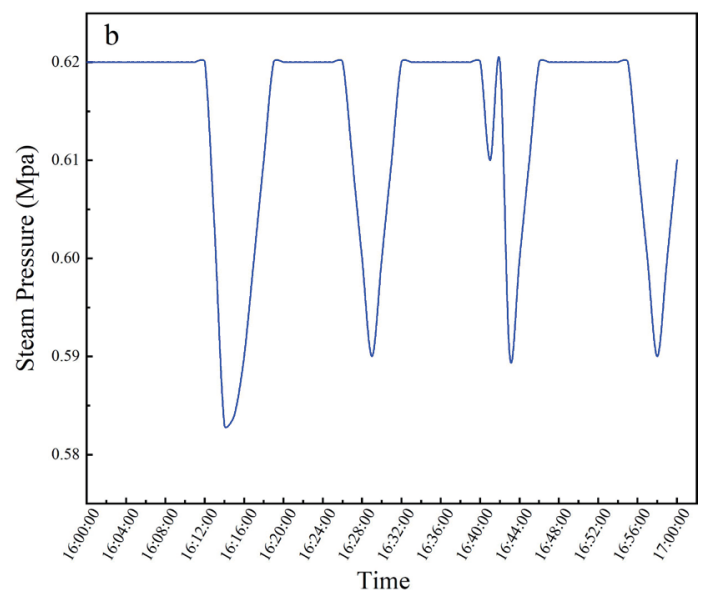

(b)

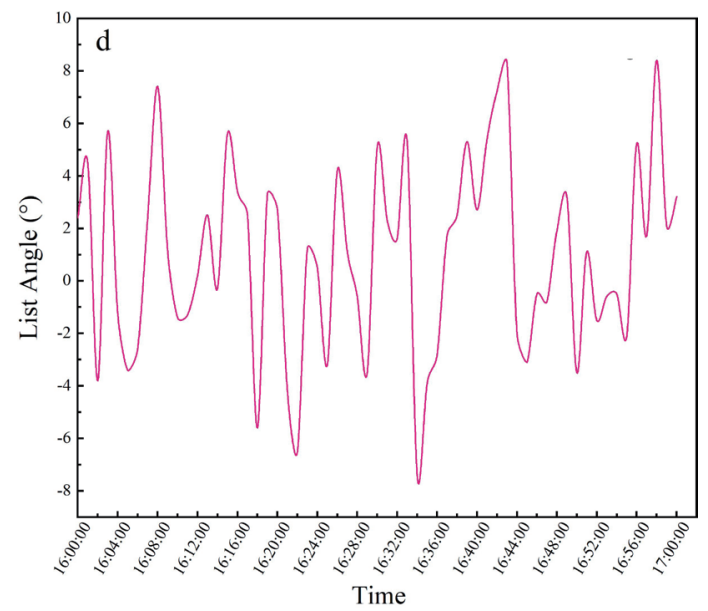

(d)

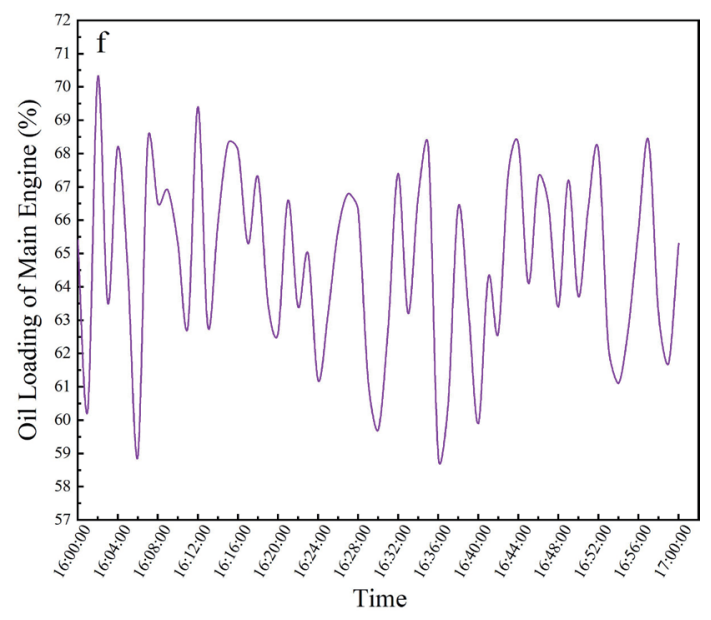

(f)

Fig. 9. (Color online) Simulation parameters and output results during ship's sailing state: (a) rudder angle, (b) boiler steam pressure, (c) boiler water level, (d) inclination angle, (e) engine speed, and (f) engine throttle load. 
and steering gear stop running, and the rudder angle, inclination angle, speed, and throttle load of the main engine are 0 . The output of the test results is basically consistent with the actual situation of Yude, showing that the proposed system can realize real-time monitoring of the ship's safety status and also provide a data reference for shore-based staff for effective management and emergency rescue.

\subsection{Discussion of test results of data transmission on board ship}

Real-time monitoring of a ship's systems is crucial to ensure the efficient and safe operation of any large carrier, which becomes especially important when multiple ships are located over a wide area. Main ship monitoring sites and operators require real-time information about all ships in an area in order to safely advise and provide proper navigation and trajectory planning. The capture and transmission of all this information is costly using current methods and not sustainable in the long run. As shown in Fig. 8, we are able to monitor simulated readings at 4 min intervals and clearly comprehend the current situation of a ship during the berthing process from the pressure levels and water levels at 4 min intervals. This information snapshot shows an hour of precise ship system monitoring useful for historical analysis and tracking.

Figure 9 shows the simulation output results of the rudder angle, boiler steam pressure, boiler water level, inclination angle, engine speed, and engine throttle load. An operator is able to use this low-cost, reliable, and secure output information when a ship is in motion and adjust its ship's angle and engine output. Consistent real-time output of this ship information can give a clear picture of a ship's status and movement at all times. In the event of a disaster or a mishap, this information provides concrete data of a particular ship at 4 min intervals, essentially similar to an airplane's 'black box', which shows a record of events, that is broadcast to nearby ships or a main operator.

\section{Conclusions}

In this article, we provided a thorough analysis of the technical characteristics of several common means of remote data transmission. By selecting the high-precision positioning, navigation, timing, and short message communication functions of BDS, we designed and developed a BDS-based data transceiver and shore-based ship data reception procedures. Our test results achieved our desired outcome. The BDS-based remote transmission system has a simple structure, which is reliable, stable, low cost, and safe. This system enables the remote monitoring and management of ships at sea, and is beneficial for maritime navigation safety and maritime maintenance in China's sea areas. The rescue, mitigation, and possible prevention of maritime accidents are positively reinforced by this system. In our future work, the chip in this system can be replaced with higher performing chips to further enhance system portability, and enable video/image transmission as the bandwidth limitation of the BeiDou module improves. 


\section{References}

1 Y. L. Zhu, G. Q. Zhang, G. N. Qiao, and L. Zhu: 7th Int. Conf. Wireless Communications, Networking and Mobile Computing (IEEE, 2011) 1. https://doi.org/10.1109/wicom.2011.6040250

2 X. Y. Kang: China. New. Telecommun. 5 (2013) 67. https://doi.org/10.3969/j.issn.1673-4866.2013.05.044

3 Y. Wang, Z. M. Zeng, Y. B. Li, W. Zhang, and H. Fang: Adv. Mater. Res. 1079 (2014) 752. https://doi. org/10.4028/www.scientific.net/AMR.1079-1080.752

4 J. M. Xia, S. J. Zhang, D. R. Xiao, and J. Oswaldo: Internat. J. Digit. Content. Tech. Appl. 14 (2012) 8. https:// doi.org/10.4156/jdcta

5 B. Qu, J. B. Nie, M. X. Shen, and K. Wang: Adv. Mater. Res. 945 (2014) 2703. https://doi.org/10.4028/www. scientific.net/AMR.945-949.2703

6 H. H. Wang, X. G. Tuo, Y. Li, Q. Lin, D. L. Nie, L. Y. Meng, and J. X. Yang: Procedia Comput. Sci. 107 (2017) 111. https://doi.org/10.1016/j.procs.2017.03.065

7 K. Su, S. G. Jin, and G. Q. Jiao: Meas. Sci. Tech. 31 (2020) 13. https://doi.org/10.1088/1361-6501/ab69d5

8 A. H. H. Kim, E. D. Nilsson, K. Radovan, E. M. Mårtensson, E. Mikael, Å. Hagström, and G. Leeuw: J. Geophys. Res. (2010). https://doi.org/10.1029/2009JD012522

9 China Satellite Navigation Office: Development of the BeiDou Navigation Satellite System (Version 4.0) (CSNO, 2019). http://www.beidou.gov.cn/xt/gfxz/201812/P020181227529626058961.pdf

10 S. B. Chandanapalli: J. Aquacult. Res. Dev. 5 (2014) 1. https://doi.org/10.4172/2155-9546.1000283

11 S. A. Ren, H. W. Chen, H. P. Zhang, Z. Liu, and S. S. Wu: Adv. Intell. Syst. Res. (2015). https://doi.org/10.2991/ icismme-15.2015.329

12 J. W. Zhan, H. B. Tong, H. Sha, G. Z. Zhang, and G. Ou: Proc. China Satellite Navigation Conf. (CSNC, 2012) 215-225. https://doi.org/10.1007/978-3-642-29193-7_20

13 G. Edgar: Marine Policy 10 (1986) 185. https://doi.org/10.1016/0308-597X(86)90051-5 